\title{
12. ERKRANKUNGEN VON BRONCHIEN, LUNGE, PLEURA
}

\subsection{TRACHEALRUPTUR}

Symptome

Entwicklung eines Hautemphysems an Gesicht, Hals, Thorax

Dyspnoe, Zyanose, Hämoptyse

Krepitation

Rö: Pneumothorax, Mediastinalemphysem,

Weichteilemphysem

Diagnose: Klinik-Hautemphysem, Thoraxröntgen,

Tracheoskopie

\section{Therapie:}

Operative Versorgung

\subsection{CHRONISCH OBSTRUKTIVE LUNGENERKRANKUNG (COPD)}

Chronische obstruktive Bronchitis Lungenemphysem

\subsubsection{CHRONISCH OBSTRUKTIVE BRONCHITIS}

Klinische Symptomatik: Husten mit Auswurf, Belastungsdyspnoe, rezidivierende Infekte, Leistungsabfall (Worth et al., 2002)

Diagnose: Anamnese, Auskultation: Giemen, Brummen, Lungenfunktion: erhöhter Strömungswiderstand, FEV1 vermindert, $\mathrm{BGA}: \mathrm{pO}_{2}$-reduziert, $\mathrm{pCO}_{2}$ erhöht, Sputum-Bakteriologie
Therapie:

- Beseitigung der auslösenden Noxe

- Atemtherapie

- Sauerstoffapplikation

- Sanierung von Infekten

- Medikamentöse antiobstruktive Therapie:

- Lang wirksames inhalatives $\beta 2$-Sympathomimeticum

- Lang wirksames orales Theophyllinpräparat

- Orales Glukokortikoid im akuten Stadium - dann ausschleichende Dosierung

\subsubsection{LUNGENEMPHYSEM}

Klinisch können zwei unterschiedliche Typen von Emphysematikern differenziert werden:

Pink puffer: hager, deutliche Dyspnoe, geringe Zyanose, trockener Husten

Blue bloater: Übergewicht, kaum Dyspnoe, deutliche Zyanose, produktiver Husten

Diagnose: Auskultation: silent lung, Perkussion: hypersonorer Klopfschall, $\mathrm{BGA}: \mathrm{pO}_{2}$ vermindert, $\mathrm{pCO}_{2}$ erhöht, Lungenfunktion: erhöhtes intrathorakales Gasvolumen, Widerstände erhöht bei Obstruktion, FEV1 vermindert

Labor: $\alpha 1$-Antitrypsinmangel 


\section{Therapie: konservativ}

- Atemtherapie - nicht invasive Beatmung

- Sanierung von Infekten

- Sauerstoffapplikation, vorsichtig - Cave: Bei Bestehen einer Globalinsuffizienz ist die Hypoxie der einzige Atemantrieb

Medikamentöse antiobstruktive Therapie:

- Inhalative längenwirksame $\beta 2$-Sympathomimetika

- Orale Theophyllinpräparate

- Orales Glukokortikoid

- Invasive Beatmung bei respiratorischer Dekompensation

- $\alpha 1$-Antitrypsinkonzentrat bei $\alpha 1$-Antitrypsinmangel

\section{Operative Therapie:}

- Lungenvolumsreduktion

- Lungentransplantation

\subsubsection{ASTHMA BRONCHIALE}

Klinische Symptomatik: Anfallsweise auftretende Atemnot, exspiratorischer Stridor, verlängertes Exspirium, Husten, Verwendung der Atemhilfsmuskulatur, Tachykardie, Zyanose

Diagnose: Anamnese, trockene Rasselgeräusche wie Giemen und Brummen, Lungenfunktion: vermindertes FEV1, nach Gabe eines $\beta 2$-Sympathomimeticum Steigerung der FEV1 (20 \%)

Komplikationen: Status asthmaticus, respiratorische Insuffizienz, Cor pulmonale, pulmonale Hypertonie

\section{Therapie:}

- Medikamentöse antiobstruktive Therapie: kurzwirksame $\beta 2$-Sympathomimetica

- Theophyllin i.v.

- Inhalative Kortikoide

- Behandlung von Infekten

- Allergenelimination

\subsubsection{STATUS ASTHMATICUS}

Diagnose: Giemen und Pfeifen über der Lunge, Dyspnoe, Unruhe, bei Hypoxie Verwirrtheit

Blutgase: $\mathrm{PaO}_{2}$ Abfall $<50 \mathrm{~mm} \mathrm{Hg}$, Hyperkapnie

Therapie:

- $\beta 2$-Sympathicomimetica Epinephrin (ADRENALIN $^{\circledR}$ ), Terbutalin (BRICANYL ${ }^{\circledR}$ )

- Phosphodiesterasehemmer Theophyllin (EUPHYLLIN ${ }^{\circledR}$, RESPICUR $^{\circledR}$ ) 0,24 g $1 \mathrm{~A}$ i.v. dann 3A $250 \mathrm{ml}$ kontinuierlich

- Glukokortikoide Prednisolon SOLU-DACORTIN ${ }^{\circledR} 250 \mathrm{mg}$ i.v.

- Atemunterstützung: $\mathrm{O}_{2}$-Zufuhr - Nicht invasive Beatmung (Ram et al., 2005), Masken, ASB, bei drastischer Verschlechterung des Gasaustausches - Intubation, Relaxierung kontrollierte Beatmung

- Sedierung: Benzodiazepine (muskelrelaxierende Effekte) nur bei Beatmungsmöglichkeit z. B. Midazolam - DORMICUM ${ }^{\circledR}$, Piritramid (DIPIDOLOR ${ }^{\circledR}$ ) - Atemdepression, Propofol (DIPRIVAN $^{\circledR}$ ), Ketanest $\left(\right.$ KETANEST $^{\circledR}$ ) bei Beatmung

- 32-Sympathicomimetica - repititive Inhalation

- Kurz wirksame $\beta$-2-Sympathomimetica: Wirkdauer 4-6 Stunden

- Fenoterol- BEROTEC ${ }^{\circledR}$, SalbutamolSULTANOL ${ }^{\circledR}$, Terbutalin-BRICANYL ${ }^{\circledR}$

- Langwirksame $\beta$-Sympathomimetica: Wirkdauer 8-12 Stunden, nicht geeignet zur Soforttherapie des Asthmaanfalles

- Methylxanthine (Theophyllin-Derivate)

- Parasympatholytica (Anticholinergica)

- Ipratropiumbromid (Inhalation) ATROVENT ${ }^{\circledR}$

- Inhalation mit Magnesium-Sulfaten (Blitz et al., 2005)

\subsection{OBERE ATEMWGESOBSTRUKTION}

\section{Hypopharyngeale Obstruktion}

$\mathrm{Zu}$ einer Verlegung der oberen Atemwege kann es durch Fremdkörper, erbrochene Nahrungsreste und Blut kommen. Zusätzlich können Angioödeme wie Quincke-Ödeme zu einer Verlegung der oberen Atemwege führen. 
Therapie: Inspektion unter Narkosebereitschaft, Tubus, Magillzange

\section{Stridor}

Meist postoperativ auftretender geringfügiger Stimmlippenkrampf, der in der Aufwachphase nach Extubation oder nach laryngealen Eingriffen auftreten kann.

\section{Therapie:}

Zunächst Gabe eines Hypnotikums-Propofol, assistierte Maskenbeatmung, wenn der Stridor nicht besser wird eventuell Inspektion mit einem Laryngoskop und Absaugen im Larynxbereich unter Sicht.

\section{Laryngospasmus}

Massiver Stimmlippenkrampf.

Ein Laryngospasmus kann nicht durch eine gewaltsame Intubation beseitigt werden, da es nicht möglich ist, die Glottisebene mit einem Tubus zu passieren. Ebenso gelingt es nicht oder kaum, Luft mittels Maskenbeatmung in die Lunge zu bringen.

\section{Therapie:}

Relaxierung mit einem kurz wirksamen Muskelrelaxans, ebenso Gabe eines Hypnotikums, neuerliche Entwöhnung mittels Maskenbeatmung

\subsection{ATEMWEGSBRAND}

\section{Auftreten}

Unfallbedingt

Im medizinischen Bereich bei operativen Eingriffen in den Atemwegen mit dem Laser $\left(\mathrm{CO}_{2}\right.$-Laser $)$.

\section{Vorgehen}

1. Sofortige Entfernung des brennenden oder glosenden Endotrachealtubus

2. Lokales Ablöschen von etwaig brennendem OPMaterial

3. Beendigung der Beatmung

4. Wiederaufnahme der Beatmung (ev. Re-Intubation)

5. Beurteilung des Hitzeschadens durch Bronchoskopie

\section{Therapie:}

Steroide, Antibiotika

Ev. Tracheotomie

Intensivüberwachung

\subsection{ASPIRATION}

Fast immer Mischinfektion von aeroben Keimen (Staphylokokken, Enterobakterien, Pseudomonas Arten) und anaeroben Keimen (Fusobakterien, Bakteroides, Peptostreptokokkus)

Diagnose: Lungenröntgen, Bronchoskopie

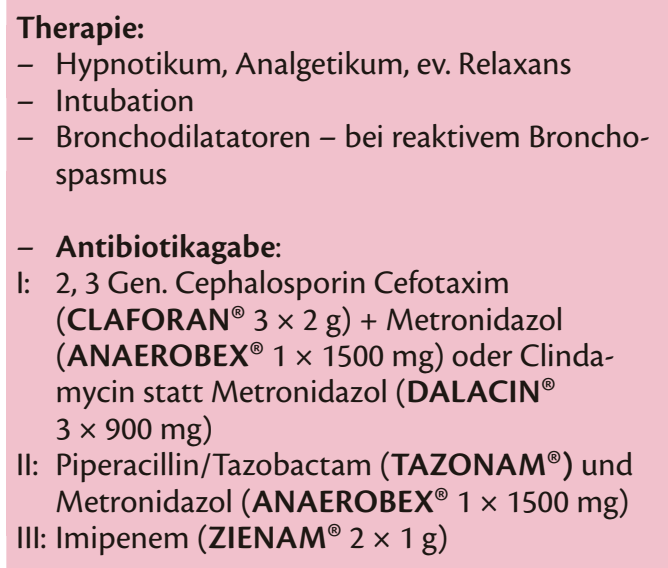

\subsection{PLEURAERGUSS}

Pleurale Flüssigkeitsansammlungen sind bei einer Vielzahl pulmonaler wie extrapulmonaler Erkrankungen zu finden (Georg RB, 1995). Bei Bestehen eines Pleuraergusses muss geklärt werden, ob es sich um eine Transudat oder eine Exsudat handelt. Das Transudat ist definiert als ein nichtentzündlicher Erguss, der aufgrund hydrostatischer oder onkotischer Druckdifferenzen entsteht oder auch durch eine Flüssigkeitsverschiebung aus dem peritonealen Raum. Bei einem Exudat handelt es sich um ein Entzündungsgeschehen der Pleura mit konsekutiver Beeinträchtigung der Lymphdrainage aus dem Pleuralraum.

Radiologisch liegt die untere Nachweisgrenze zwischen 100 und $300 \mathrm{ml}$, sonografisch jedoch bei Mengen über $10 \mathrm{ml}$. 

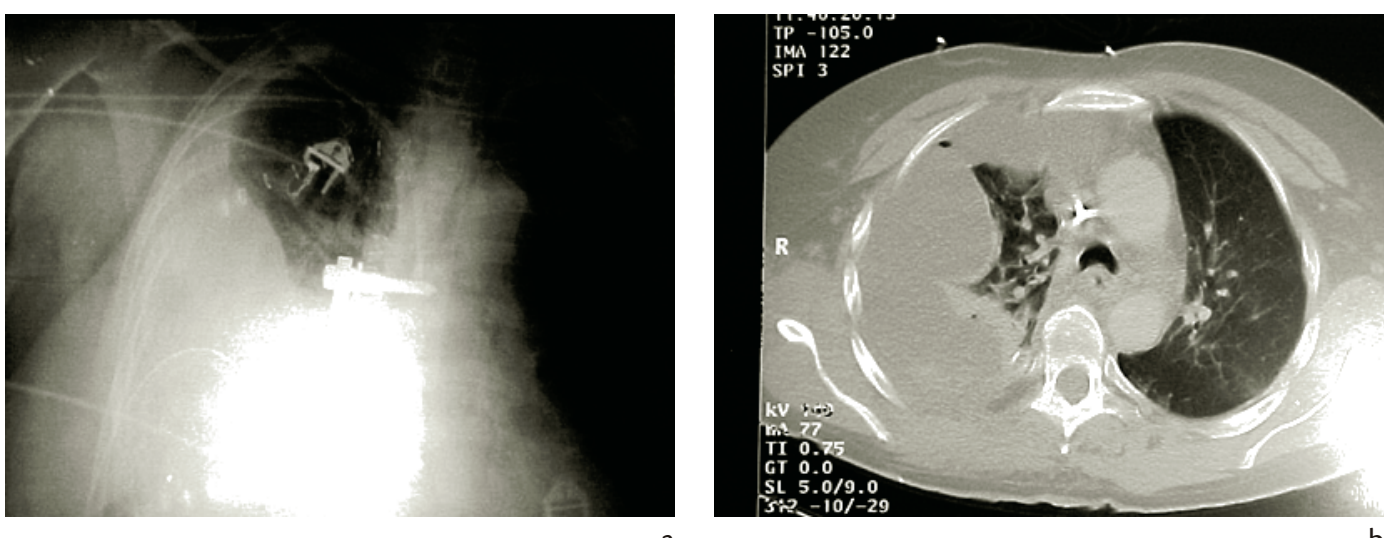

Abb. 66. (a) Pat. W. M. Verschattung thorakal. (b) Pat. W. M. Thorax-CT ebenfalls postoperativ, ausgedehnte Verschattung rechts. DD: Erguss, Hämatom

Die Pleurapunktion muss am Oberrand der Rippe durchgeführt werden, wobei bei größeren Ergüssen nicht mehr als $1000 \mathrm{ml}$ punktiert werden sollten. Gefahr der Ausbildung eines reaktiven akuten Lungenödems durch schnelle Erhöhung der Vaskularisation der vorher kollabierten Alveolen und ansteigende Kapillarpermeabilität.

Differentialdiagnose Exudat/Transudat anhand der Light-Kriterien (Light et al., 1972; Light, 1995).

Vorliegen eines Exudates, wenn mindestens eines der genannten Kriterien vorliegt:

1. Quotient Gesamteiweiß - Erguss/Serum > 0,5

2. Quotient LDH - Erguss/Serum $>0,6$

3. LDH-Gehalt im Erguss ist größer als zwei Drittel des oberen Normwertes des Serum-LDH-Gehaltes.

Ein anderer Parameter ist der Cholesteringehalt, der im Transudat $<60 \mathrm{mg} / \mathrm{dl}$, im Exudat $>60 \mathrm{mg} / \mathrm{dl}$ ist, der Eiweißgehalt ist bei einem Transudat $<3,0 \mathrm{~g} /$ $100 \mathrm{ml}$ und bei einem Exudat > 3,0 g/100 ml.

Transudat

Herzinsuffizienz

Leberzirrhose

Perikarditis

Nephrotisches Syndrom

Myxödem

Lungenembolie

Hypalbuminämie $<25 \mathrm{~g} / \mathrm{l}$
Exudat

Infektionen

- bakterielle Infektionen

- Tuberkulose

- Pilzinfektionen

- virale Infektion

- parasitäre Infektion

Neoplastische Erkrankung

Mesotheliom, metast. Erkrankung

Gastrointestinale Erkrankung

- akute Pankreatitis

- Ösophagusperforation

- intraabdomineller Abzess

Hämothorax

Chylothorax

- traumatisch, nicht traumatisch

Medikamentenüberempfindlichkeit

- Dantrolene

- Nitrofurantoin

- Methysergid

- Amiodarone

- Bromocriptin

\subsection{CHYLOTHORAX (Abb. 66 a, b)}

Genese: (De Beer et al., 2000, Terzi et al., 1994) traumatisch oder nicht traumatisch

Diagnose: typisch ist ein Triglyzeridspiegel $>110 \mathrm{mg} /$ dl; Triglyzeridspiegel $<50$ mg/dl schließt diese Diagnose aus 
Größere intrathorakale Chylusansammlungen lassen sich, da Chylus strahlendicht ist, im Lungenröntgen erkennen. Im frontalen ant. - post. Strahlengang Verwechslung mit Atelektase oder intrathorak. Haematom möglich. Seitenbild: typischerweise Flüssigkeitsansammlung im Mediastinum posterius.

Pat. W. M. Laborbefunde

$\begin{array}{lll}\text { Chylus } & \text { Pleura rechts } & \text { Serum } \\ \text { Triglyzeride } & 282 \mathrm{mg} / \mathrm{dl} & 95 \mathrm{mg} / \mathrm{dl} \\ \text { Cholesterin } & 28 \mathrm{mg} / \mathrm{dl} & 135 \mathrm{mg} / \mathrm{dl} \\ \text { Potein ges. } & 14,1 \mathrm{~g} / \mathrm{l} & 47,9 \mathrm{~g} / \mathrm{l} \\ \text { Albumin } & 8,9 \mathrm{~g} / \mathrm{l} & 25,3 \mathrm{~g} / \mathrm{l} \\ \mathrm{Na} & 149 \mathrm{mmol} / \mathrm{l} & 136 \mathrm{mmol} / \mathrm{l} \\ \mathrm{K} & 4,4 \mathrm{mmol} / \mathrm{l} & 4,4 \mathrm{mmol} / / \\ \mathrm{Cl} & 113 \mathrm{mmol} / \mathrm{l} & 109 \mathrm{mmol} / \mathrm{l}\end{array}$

Gesamtmenge täglich, welche über 2 Thoraxdrainagen gefördert wurde: $5200 \mathrm{ml}$

\section{Therapie:}

1. Thoraxdrainage zur Entleerung des Chylus

2. Intravenöse Hyperalimentation

3. Wenn der Patient essen darf ist eine:

a. enterale Diät ohne jegliche Fetternährung

b. enterale Diät mit mittelkettigen Triglyzeriden möglich

b 1. Isosource MCT

b 2. Fresubin $M C T$

4. Inhalation von NO $20 \mathrm{ppm}$ (Berkenbosch et al., 1999)

5. Ligatur des d. thoracicus (nach Auffinden desselben)

Wenn die Drainage mehr als $1500 \mathrm{ml} /$ die Chylus fördert und zu Ernährungsproblemen (Katabolie) führt oder mehr als 28 Tage besteht, sollte eine chirurgische Intervention notwendig sein. (Ligatur des duct. thoracicus)

Bei nicht traumatischem Chylothorax besteht die Möglichkeit der Anlegung eines pleuro-peritonealen Shuntes.

\section{$=\quad$ PSEUDOCHYLOTHORAX CHYLIFORM EFFUSION}

Stellt eine Effusion von Flüssigkeit dar, mit einem hohen Gehalt an Cholesterin. Hingegen sind Triglyzeride oder Chylomikronen nicht vorhanden und dieses Zustandsbild hat nichts mit dem lymphatischen Gefäßsystem oder Chylus zu tun.

Triglyzeride n.v.

Cholesterinspiegel $>200 \mathrm{mg} / \mathrm{dl}$

\subsection{LUNGENBLUTUNG}

Ursache: Gerinnungsstörung +Arrosion, Infarkt, Tumor, iatrogen, usw.

Therapie:

- lokal Vasokonstriktoren, Epinephrin ADRENALIN $^{\circledR}$

- Systemisch: Tranexamsäure - Antifibrinolyticum (bei hyperfibrinolytischer Blutung): CYCLOCAPRON $^{\circledR}$ i.v.

- Eventuell einseitige Intubation mit Doppellumentubus (nur vorübergehende Möglichkeit)

- Selektive Stillung mit starrem Bronchoskop

\subsection{LUNGENABSZESS}

Ätiologie: Ursachen eines Lungenabszesses können sein:

Entzündlich: eitrige Bronchitis, Pneumonie, Fortleitung eines entzündlichen Geschehen des Mediastinums, der Pleura oder des Zwerchfelles (subphrenischer Abszess), hämatogene Streuung eines eitrigen Entzündungsherdes

Traumatisch: Keimeinschleppung durch ein perforierendes Thoraxtrauma

Neoplastisch: durch ein zerfallendes Bronchialkarzinom

Immunsuppressiv bedingt: nach Transplantation, Drogenabusus, HIV/AIDS-Patienten, Tumorkachexie

Klinik: Beim kontaktfähigen Patienten: Husten, Dyspnoe, atemabhängige Schmerzen, Fieber, Schüttelfrost, ausgeprägtes Krankheitsgefühl 
Komplikationen: Abzessstreuung in andere Organe, Superinfektion mit Aspergillus oder Fäulniserregern wie Proteus, Pseudomonas, Anaerobier, Bronchusfistel, Pleuraempyem bei Abszessperforation in die Pleurahöhle

Diagnose: Röntgenthorax in zwei Ebenen: Abszesshöhle mit Spiegelbildung, CT-Thorax, Bronchial - Lavage

\section{Konservative Therapie:}

- Antibiotika

- CT-gesteuerte Punktion und Abszessdrainage

- Bronchoskopische Abszessdrainage

- Hyperbare Oxygenation

Operative Therapie:

- Resektion - Segmentresektion

\subsection{BRONCHIOLITIS OBLITERANS MIT ORGANISIERENDER PNEUMONIE (BOOP)}

Definition: Die konstriktive Bronchiolitis ist eine obstruktive Atemwegserkrankung, die sekundäre Bronchiolitis obliterans kommt bei Virus- oder Mykoplasmen-Pneumonien, nach Inhalation toxischer Gase, bei Kollagenosen/Vaskulitis, nach Transplantationen, als medikamentöse-toxische Reaktion vor. Unter der Krankheit „BOOP“ versteht man die idiopathische Form der Bronchiolitis obliterans, die mit einer interstitiellen, fibrosierenden Lungenerkrankung (organisierende Pneumonie) einhergeht.

Klinik: In der Klinik zeichnet sich die Symptomatik dieser Patienten oft durch ein subakutes Krankheitsbild aus, welches charakterisiert ist durch Grippegefühl mit Husten, Dyspnoe und konstitutionelle Symptome. Meistens ist der Verlauf langwierig jedoch prognostisch günstig. Beschrieben sind jedoch auch akute lebensbedrohliche Formen einhergehend mit einem adult respiratory distress syndrom.

\section{Epidemiologie, Ätiologie: Unbekannt}

Pathogenese: Unbekannt; polypoide Proliferation von myxoidem Gewebe in den terminalen Bronchiolen, die kleiner als $2 \mathrm{~mm}$ sind, und chronische interstitielle Entzündung der Alveolarwände
Differentialdiagnose: Andere chronische Pneumonieverläufe und interstitielle Lungenerkrankungen

Diagnose: Klinisches Bild der „Wanderpneumonie“ oder der chronischen Pneumonie. Offene Lungenbiopsie mit Histologie, die BAL ist unspezifisch

\section{Therapie:}

- Glukokortikoide; 1 mg/kg KG/Tag über mehrere Wochen

- Makrolidantibiotika (Stover et al., 2005, Crowley et al., 2005)

\subsection{BRONCHOPLEURALE FISTEL}

Definition: Bestehen einer Verbindung zwischen Bronchialbaum und der Pleurahöhle

Ätiologie: chirurgisches Trauma nach Lobektomie oder Pneumonektomie, als Folge einer Infektion wie Tuberkulose, Pneumonie, Empyem oder Lungenabszess, offene Thoraxverletzung.

Das Auftreten einer bronchopleuralen Fistel stellt vor allem dann ein Problem dar, wenn der Patient beatmet ist und das dazu notwendige Tidalvolumen vor allem in den pleuralen Raum abgegeben wird und nicht intrapulmonal in den Alveolarraum. Gefahr der Entwicklung eines Spannungspneumothorax.

\section{Therapie:}

- Buleaudrainage: Bei Auftreten eines Pneumothorax ist die Platzierung einer Buleaudrianage notwendig.

- Doppellumentubus - seitengetrennte Beatmung: Bei beatmeten Patienten mit massivem Gas-Volumsverlust bietet sich die Beatmung mit einem Doppellumen-Tubus und anschließender Durchführung einer seitengetrennten Beatmung an.

- High-Frequency-Jet-Ventilation (Campbell et al., 2000, Ha et al., 2005) für große bronchopleurale Fisteln. Bei bestehender Unmöglichkeit der Verabreichung ausreichender Tidalvolumina unter konventioneller Beatmung ermöglicht es eine Hochfrequenzjetventilation, aufgrund ihres hohen Gasflusses, ein ausreichendes Tidalvolumen 
trotz des Gasverlustes über die Fistel sicherzustellen. Bei Verwendung einer Combined High-Frequency-Jet-Ventilation kann mit dem oberen Druckplateau die $\mathrm{CO}_{2}$-Elimination gesteuert werden.

\subsection{AKUTE MEDIASTINITIS}

Definition: Bakterielle bzw. nicht bakterielle Entzündung des Bindegewebes im Mediastinum

Ätiologie: Infektionen im Kopf/Hals Bereich (z.B. Tonsillitis, Otitis media, Zahninfektion) Ösophagusperforation nach endoskopischem Eingriff, $\mathrm{Er}$ brechen (Boerhaave-Syndrom), nekrotisierende Entzündung, Säure/Laugenverletzung, Verletzung von Trachea und Bronchialsystem (z.B. Tumor, Bronchoskopie, Tracheotomie), Trauma, postoperativ nach Herz-Thorax-Operationen, Fortleitung einer Infektion von anderen Körperbereichen, Wirbelkörper, paraspinaler Abszeß, Sternumosteomyelitis, Halswirbelsäulenoperation (ventrale Fusion)

Klinik: Hohes Fieber, retrosternale Schmerzen und Schluckbeschwerden, Schüttelfrost, Tachypnoe, Tachycardie

Diagnose: Klinisch lässt sich ein druckschmerzhaftes Sternum, eine diskrete Schwellung der Supraklavikularregion, ev. eine Hautemphysem feststellen. Typisch für ein ausgeprägtes Mediastinalemphysem ist eine herzschlagsynchrones Knistern (Hamann-Zeichen). Eventuell findet sich bei einer ausgeprägten Infektion eine Trachealverlagerung und eine obere Einflussstauung.

Thoraxröntgen: Mediastinalverbreiterung, ev. besteht eine Pneumothorax bzw. ein Seropneumothorax

Thorax CT: die Diagnose als auch Ausdehnung kann besser festgestellt werden

\section{Erregerspektrum}

Deszendierende Mediastinitis: häufig polymikrobiell, fakultativ anaerobe Erreger: Staphylococcus, Streptococcus spezies, Peptostreptococcus, Bacteroides, Fusobacterium, Pseudomonas aeruginosa Postoperative Mediastinitis: S. aureus und S. epidermidis, (40-80\%), gram-negative Erreger und polymikrobielle Erreger bei 25-40 \%
Anaerobier besonders nach Ösophagusperforation, deszendierendem Zahnabszeß und retropharyngealem Abszeß

Komplikationen: Candidamediastinitis (Clancy C) et al., 1997 ) mit hämatogener Streuung (Letalität $56 \%$ ), direkte Ausbreitung einer Infektion zu Herz, Gefäßen, Lunge, Knochen

Prognose: Letalität der postoperativen Mediastinitis: $25 \%-52 \%$ (Lutwick et al., 1998)

Letalität der deszendierenden nekrotisierenden Mediastinitis $19 \%-47 \%$

\section{Therapie:}

- Chirurgische Sanierung eines Focus, ev. operative Exploration mit Debridement und Irrigation

- Antibiotica (Staphylokokken wirksame Antibioticatherapie) z. B. Penicillinaseresistentes Penicillin + Aminoglykosid, Carbapeneme, Chinolone

- Hyperbare Oxygenation

\section{LITERATUR}

Berkenbosch JW, Withington DE, Management of postoperative chylothorax with nitric oxide: a case report. Crit Care Med 27 (5): 1022-1024: 1999

Blitz M, Blitz S, Beasely $R$ et al., Inhaled magnesium sulfate in the treatment of acute asthma. Conchrane Database Syst Rev 19: October 2005

Campbell D, Steinmann M, Porayko L, Nitric oxide and high frequency jet ventilation in a patient with bilateral bronchopleural fistulae and ARDS. Can J Anaesth Jan 47 (1): 53-57: January 2000

Crowley S, Egan JJ, Macrolide antibiotics and bronchiolitis obliterans following lung transplantation. Expert Rev Anti Infect Ther 3 (6): 923-930: December 2005

De Beer HG, Mol MJ, Janssen JP, Chylothorax: Neth J Med 56 (1): 25-31: January 2000

George RB, chest medicine, essentials of pulmonary and critical care medicine 518: 1995

Ha DV, Johnson D, High frequency oscillatory ventilation in the management of a high output bronchopleural fistula: a case report. Can J Anaesth 51 (1): 78-83: January 2004

Light RW, Rodriguez RM, Pleural effusion: to the diagnostic separation of transudatos and exudatos. Ann Intern Med 77: 507-513

Ram Fs, Wellington S, Rowe B, Wedzicha JA, Non-invasive positive pressure ventilation for treatment of respiratory failure due to severe acute exacerbations of asthma. Conchrane Database Syst Rev 20: July 2005 
Stover DE, Mangino D, Macrolides: a treatment alternative for bronchiolitis obliterans organizing pneumonia? Chest 128 (5): 3611-3617: November 2005

Terzi A, Furlan G, Magnanelli G, Terrini A, Ivic N, Chylothorax after pleuro-pulmonary surgery: a rare but unavoidable complication. Thorac Cardiovasc Surg 42 (2): 81-84: April 1994
Worth $\mathrm{H}$ et al., Leitlinie der Deutschen Atemwegsliga und der Deutschen Gesellschaft für Pneumologie zur Diagnostik und Therapie von Patienten mit chronisch obstruktiver Bronchitis und Lungenemphysem. Pneumologie 56: 704-738: 2002 